\title{
Presidentes frente a cancillerías: la formulación de la política exterior en Brasil y Chile entre 1990 y 2010
}

\author{
Presidents versus foreign offices: The formulation \\ of foreign policy in Brazil and Chile between 1990 and 2010
}

\author{
PABLO VALENZUELA
}

Universidad de Santiago de Chile

\section{Cómo citar/Citation}

Valenzuela, P. (2019). Presidentes frente a cancillerías: la formulación de la política exterior en Brasil y Chile entre 1990 y 2010. Revista Española de Ciencia Política, 49, 63-83. Doi: https://doi.org/10.21308/recp.49.03

\section{Resumen}

El objetivo de este artículo es explicar el diferente papel e influencia de los ministerios de relaciones exteriores (o cancillerías) de Brasil y Chile durante la formulación e implementación de la política exterior. Para ello, se examina un caso relevante en cada país, aplicando un modelo de análisis basado en la política burocrática. Tres son las dimensiones a las que se presta especial atención: la mayor o menor capacidad de estos ministerios de centralizar y coordinar la agenda de política internacional; su grado de integración o aislamiento frente al resto de la administración pública, y la profesionalización del servicio diplomático. Se concluye que las dos primeras son las variables explicativas principales, si bien la tercera puede potenciar a las anteriores. La aplicación de este marco explicativo al estudio comparado de los ejemplos muestra interesantes contrastes en el modo en que Chile y Brasil formulan sus respectivas políticas exteriores.

Palabras clave: ministerios, política exterior, análisis de política exterior, Brasil, Chile.

\begin{abstract}
The purpose of this article is to explain the different role and influence exercised by Brazil's and Chile's Foreign Offices (or Chancelleries) during the formulation and implementation of their foreign policy. To this aim, it examines one relevant case in each country, applying a model of analysis based on the bureaucratic politics. Particular attention is paid to three dimensions: these ministries' greater or lesser capacity to centralize and coordinate the international policy agenda; their degree of integration with or isolation from the rest of the public administration,
\end{abstract}


and the professionalization of the diplomatic service. It concludes that the first two are the most relevant explanatory variables, while the third dimension can also boost the former ones. The application of this explanatory framework to the comparative analysis of both cases shows interesting contrasts in the way that Chile and Brazil formulate their respective foreign policies.

Keywords: ministries, foreign policy, foreign policy analysis, Brazil, Chile.

\section{INTRODUCCIÓN}

Estudiar la política exterior de los Estados implica considerar los procesos de formulación y toma de decisiones, los actores involucrados y las variables de carácter doméstico e internacional que influyen. Así definida, la formulación de la política exterior se produce por un conjunto de actores con agendas en pugna que buscan que los líderes adopten sus preferencias de política pública.

El campo del análisis de la política exterior busca explicar las razones por las que los Estados emprenden determinadas acciones en el sistema internacional usando como variables explicativas los actores domésticos, sus decisiones, comportamiento y la forma en la que perciben el mundo. La investigación se ha enfocado en el rol de los Congresos y Parlamentos en la formulación de la política exterior (Kaarbo y Kesgin, 2010; Marshall y Prins, 2011; Mack et al., 2013), de la burocracia y los ministerios de Relaciones Exteriores (Drezner, 2000; Halperin et al., 2006; Marsh, 2013), del liderazgo de los jefes de Gobierno (Kaarbo, 1997; Kaarbo y Hermann, 1998; Keller y Foster, 2012) y, en general, de los mecanismos que permiten explicar el proceso de formulación y toma de decisiones de la política exterior (Beasley et al., 2001; Kaarbo, 2001; Hudson, 2005).

La pregunta que guía esta investigación es: ¿qué rol ha jugado el Ministerio de Relaciones Exteriores (MRE) ${ }^{1}$ de Chile y Brasil en los procesos de formulación de política exterior? Según la literatura (Pimenta de Faria et al., 2013; Casaróes, 2012; Gomes Saraiva, 2010a; Batista, 2009) sabemos que el MRE de Brasil juega un papel relevante en la formulación e implementación de la agenda de política exterior. No obstante, es menos lo que conocemos sobre el resto de los ministerios de la región. La hipótesis planteada es que en la medida que el MRE tenga mayores niveles de profesionalización, se encuentre más aislado dentro de la Administración pública y sea capaz de coordinar de forma centralizada las acciones de política exterior del Estado entre todos los actores involucrados, podrá actuar con mayor autonomía en su relación con la Presidencia de la República y, por lo tanto, tendrá una capacidad mayor para plantear

1. El trabajo usa indistintamente los términos «ministerios de relaciones exteriores» $\mathrm{y}$ «cancillería», que es la forma en la que estas agencias estatales se denominan comúnmente en América Latina. 
agendas sectoriales alternativas o complementarias a la del jefe de Gobierno, pudiendo jugar un rol relevante durante el proceso de toma de decisiones.

Se han seleccionado dos procesos de política exterior que permitirán acercarse a una decisión de similar naturaleza para analizar la configuración de los actores y el rol del MRE. El caso de Brasil se estudia a través de la posición adoptada frente a la creación de la Unión de Naciones Suramericanas (UNASUR) y, en general, la estrategia hacia Sudamérica. En el caso chileno se ha considerado el proceso de creación e incorporación a la Alianza del Pacífico y la estrategia comercial del país. Para estudiar estos procesos de formulación de política exterior, se utiliza el modelo de bureaucratic politics (Welch, 1992; Preston y 't Hart, 1999; Drezner, 2000; Halperin et al., 2006; Carlsnaes, 2012; Marsh, 2013), que permite analizar el comportamiento de los actores en el marco de las organizaciones burocráticas a las que pertenecen y las agendas y los roles que asumen en la interacción con otros actores durante la formulación de la política exterior.

La segunda sección de este trabajo presenta el marco teórico utilizado para el estudio: el análisis de política exterior y el marco de análisis del modelo de bureaucratic politics. La tercera sección presenta algunos aspectos metodológicos del trabajo y, a continuación, las estrategias de política exterior seleccionadas para los casos de Chile y Brasil, la forma en que las cancillerías de ambos países han participado en la formulación de la misma y la interacción que han mantenido con otros actores. La sección cuatro presenta una breve discusión comparada en torno a la pregunta de investigación y a los factores explicativos que diferencian ambas cancillerías estudiadas. El último apartado presenta las conclusiones generales del estudio y las preguntas que pueden ser abordadas en investigaciones posteriores.

\section{EL ANÁLISIS DE POLÍTICA EXTERIOR COMO MARCO TEÓRICO}

El análisis de política exterior (FPA, foreign policy analysis) es un campo de estudios que se ubica en un punto intermedio entre las relaciones internacionales y la política comparada. Conceptualmente, dos pilares sustentan el FPA: el primero indica que lo más importante en política internacional son las decisiones que toman actores individuales o colectivos en el proceso de formulación de la política exterior desde el ambiente doméstico (Breuning, 2007: 164), mientras que el segundo señala que los decisores deben enfrentarse a limitaciones institucionales y psicológicas de carácter doméstico e internacional (Taliaferro et al., 2009).

Siguiendo a Hudson (2005), el FPA busca explicar el proceso y los resultados de la adopción de decisiones humanas con referencia a sus consecuencias en entidades internacionales. Sin embargo, también el análisis de política exterior considera los factores que influyen en quienes toman la decisión y en la decisión escogida. Hay dos características que son más propias del análisis de política exterior: primero, se trata de un análisis multifactorial que considera variables en varios niveles (Hudson, 2005: 2); segundo, el FPA permite construir una intersección entre los determinantes primarios 
del comportamiento del Estado (factores materiales e ideas) y su actuación en la arena internacional.

Desde esta perspectiva, el análisis de política exterior considera al decisor a nivel individual, sus características personales, su liderazgo y su forma de ver el mundo (Hudson, 2007; Keller y Foster, 2012) y la forma en la que otros actores influyen en ese proceso de toma de decisiones, tales como la opinión pública, los medios de comunicación, el cuerpo legislativo, el gabinete, los asesores, el poder judicial, entre otros agentes gubernamentales que no solo limitan la toma de decisiones, sino que además tienen motivaciones para buscar instalar su propia agenda (Knecht y Weatheford, 2006; Foyle, 1997).

Una de las aproximaciones para estudiar la interacción entre actores es la política burocrática, que para este trabajo resulta útil, pues considera a los individuos como integrantes de las agencias burocráticas que son parte del Estado. Dichas agencias tienen sus propias limitaciones, cultura organizacional y un rol definido dentro de la Administración pública. Eso llevaría a que quienes actúan dentro del Estado lo hagan también a partir de la posición que ocupan (Alden y Aran, 2012). Por ejemplo, Halperin et al. (2006) señalan que, si bien puede compartirse una imagen general respecto de la política internacional, los actores tienden a ver el mundo de diferentes maneras y cada uno busca que el Gobierno realice lo que ellos consideran que es lo mejor, mientras que desde la perspectiva de Alden y Aran (2012) diferentes configuraciones institucionales dan como resultado diversas miradas respecto a las acciones y decisiones que se deben emprender en política exterior.

Los actores burocráticos pueden ser los ministerios, las ramas de las Fuerzas Armadas, las instancias de comando conjunto de la defensa o aquellas formas de asesoría que estén a disposición del presidente. La Oficina de la Presidencia también se puede convertir en un actor burocrático. Así, Marsh (2013) utiliza el modelo de política burocrática como marco de análisis para explicar la decisión de aumentar las tropas en Afganistán, tomada por el presidente de los Estados Unidos en diciembre de 2009. En su estudio, Marsh incorpora a la Oficina del Secretario de Defensa, el Estado Mayor Conjunto, el Comando Central, el Consejo de Seguridad Nacional, el Departamento de Estado y la Oficina de la Presidencia (Marsh, 2013: 6-7)

La política burocrática cuestiona la concepción del rol nacional, pues explica la existencia de desacuerdos sobre las acciones que debe seguir el Estado. Se trata de preguntarse dónde reside la formulación de la política exterior: si en los intereses parroquiales de cada una de las organizaciones o en el más importante interés nacional (Preston y 't Hart, 1999: 53). Los actores, desde la perspectiva de la política burocrática, eligen no a través de un conjunto consistente de objetivos estratégicos, sino de acuerdo a varias concepciones respecto a la seguridad nacional, las características organizacionales y los intereses domésticos y personales. La decisión no es una simple elección racional, sino un proceso de pulling and hauling (Alden y Aran, 2012). Se debe considerar también que los individuos tienen un rol dentro de la burocracia, basado en expectativas y responsabilidades derivadas de su empleo, sus derechos y su esfera de autoridad, y esto implica que las estructuras burocráticas también pueden formar 
preferencias en los actores (Hollis y Smith, 1986: 285). De acuerdo con Alden y Aran (2012) los políticos y los burócratas son juzgados por el cargo que ostentan, más que por sus características personales, por lo que es plausible señalar que el rol como titular de un cargo se sobreponga, aunque no totalmente, al rol individual. Además, un cargo ocupado por un individuo determina, al menos en parte, las relaciones establecidas con otros actores que ostentan también la titularidad en otras oficinas.

Buscando influir en el proceso de toma de decisiones, los actores pueden usar estrategias diversas que dependen de los recursos de poder o las ventajas para la negociación con los que cuenten. Eventualmente podrían buscar formar coaliciones de actores dentro o fuera de la Administración, generar presiones con los medios de comunicación o distorsionar la información mostrándola de una forma favorable a sus intereses (Kaarbo, 1998: 88; Preston y 't Hart, 1999: 55). En el caso de las minorías burocráticas, por ejemplo, pueden ser verticales u horizontales (Kaarbo y Grunfeld, 1998). Las verticales se encuentran subordinadas en la jerarquía de la Administración pública y deben convencer a sus superiores, mientras que las horizontales se encuentran en el mismo nivel que otros departamentos y para poder ejercer su influencia deben enfrentarse a ellos. Esa posición dentro de la Administración determina las estrategias más útiles que pueden seguir.

Ministerios de relaciones exteriores más fuertes podrían determinar agendas de políticas exterior mucho más estables, dado que las ideas permanecen en el interior de las organizaciones burocráticas más allá de la duración de un Gobierno (Drezner, 2000). Por el contrario, equipos de cancillería menos profesionales y más dependientes del poder político serían menos capaces de mantener agendas de política exterior, por lo que la misma sería más inestable. Rosati (1981) señala que no se trata de si el jefe de Gobierno o la burocracia tiene una mayor influencia en la política exterior, sino que es mucho más útil reconocer las diferentes circunstancias a través de las cuales los actores pueden ejercer varios grados de influencia en la política exterior.

\section{EL ROL DE LA CANCILLERÍA EN LA FORMULACIÓN DE LA POLÍTICA EXTERIOR}

La variable dependiente de esta investigación es la capacidad del Ministerio de Relaciones Exteriores (MRE) para influir en la formulación de la política exterior. La primera variable independiente es la posición de la Cancillería en la Administración pública, definida como el grado de integración o aislamiento del Ministerio dentro de la Administración. Un MRE aislado podrá conservar valores organizacionales que se reflejen en las opciones de política exterior que este proponga, mientras que si se encuentra integrado podrá ser influido por la agenda de otros actores y llegará a niveles mayores de compromiso con el resto de la Administración (Drezner, 2000: 734-736; Kaarbo, 1998). La segunda variable independiente es la capacidad del Ministerio para coordinar centralizadamente la agenda de política exterior, es decir, si el MRE tiene la capacidad para coordinar las agendas de política exterior en pugna con 
otros actores y el resultado es formulado solo por el MRE, será menos probable una confrontación entre agencias y la Cancillería tendría una opción mayor para influir en el curso de política seleccionado.

La tercera variable independiente es la profesionalización del servicio exterior. Un servicio exterior profesional tiende a tener rigurosas formas de selección y formación para los diplomáticos de carrera y la mayoría de los altos cargos en las embajadas relevantes para el país son ocupados por funcionarios profesionales, sin perjuicio de que algunas embajadas puedan ser ocupadas por funcionarios políticos, con el objetivo de cumplir alguna finalidad específica (Gyngell y Wesley, 2003: 125). Un servicio exterior profesionalizado se caracteriza también por poseer áreas encargadas de proponer opciones de política exterior, pudiendo así construir su propia agenda (Gyngell y Wesley, 2003: 109).

La hipótesis es que un ministerio aislado en la Administración pública, con capacidad para coordinar centralizadamente la agenda de política exterior y que cuente con un servicio exterior profesionalizado, podrá influir de manera significativa en la en la formulación de la política exterior y servir como contrapeso al rol que puede jugar el jefe de Gobierno, en especial en presidencialismos fuertes como las latinoamericanos. En consecuencia, una variable interviniente en el modelo sería el liderazgo presidencial, definido como el grado de involucramiento del presidente en la política exterior.

La variable dependiente se define a partir de lo que proponen Preston y 't Hart para el análisis de la política burocrática dentro del poder ejecutivo (1999: 57). La política burocrática se analiza a través de tres dimensiones normativas: reality testing, acceptability y effciency. Cada una de estas dimensiones está formada por tres categorías. La reality testing la conforman sobre la simplificación burocrática, la deliberación burocrática y la distorsión burocrática. En la primera, un actor o una coalición de actores emergen como dominantes en el proceso de política y enmarcan el problema dentro de sus propias preferencias, permitiendo solo que un rango estrecho de información y argumentos entren en la discusión. La distorsión burocrática, en cambio, se refiere a que las posiciones de los actores son muy difíciles de cambiar, por lo que los argumentos de los demás actores son distorsionados impidiendo un adecuado análisis colectivo de la situación. El nivel óptimo es denominado bureaupolitical deliberation. De lo anterior se deriva una pregunta general para orientar el análisis: ¿̨tiene el Ministerio de Relaciones Exteriores la capacidad de dominar el debate respecto de la agenda de política exterior?

La segunda dimensión —acceptability— está conformada por el aislacionismo burocrático, bajo el que un actor o una coalición de actores promueven su propia agenda sin considerar el apoyo de otros actores. El otro extremo es la parálisis burocrática, en la que todos los actores defienden sus propias agendas, pero ninguna alcanza el apoyo mínimo necesario como para imponerse en la toma de decisiones. El punto óptimo es denominado por Preston y 't Hart como bureaupolitical compromise formation. La pregunta general que se deriva de esta dimensión es: ¿puede el Ministerio de Relaciones Exteriores impulsar su agenda sin el compromiso de otros actores? 
La dimensión efficiency está conformada por la categoría haste, que ocurre cuando se produce un acuerdo rápido respecto al proceso de política exterior, pero el asunto debe regresar a los decisores en etapas posteriores, con lo que se renueva la controversia, o bien sucede que por problemas de implementación el asunto debe volver a ser tratado. La categoría opuesta es waste, bajo la cual el proceso político se mantiene circulando de manera indefinida en la agenda sin producir una acción concluyente.

Con las dimensiones de Preston y 't Hart se puede construir un continuo respecto a la influencia de la Cancillería en la política exterior, que iría desde un total predominio del Ministerio respecto a otros actores o bien una total irrelevancia dada la presencia de otros actores capaces de promover sus agendas, incluyendo al presidente de la República. La posición del Ministerio en este continuo sería explicada por las tres variables independientes definidas.

\section{La politica exterior de Chile y el rol de la Cancillería}

Desde el retorno a la democracia, la política exterior de Chile ha estado marcada por la búsqueda de acuerdos de libre comercio y la promoción de las exportaciones, lo que ha generado un alejamiento, al menos parcial, de las instituciones de la región que promueven valores diferentes, tales como Mercosur o la Comunidad Andina de Naciones (CAN). Esta estrategia de regionalismo abierto implica que América Latina está presente, pero no de forma exclusiva. Por esa razón, Chile ha buscado insertarse también en otros contextos regionales como fue el NAFTA, la APEC y la Unión Europea o de forma bilateral mediante acuerdos particulares como sucede con México o Canadá (Wilhelmy y Durán, 2003: 281-282).

Varios autores (Wilhelmy y Lazo 1997; Milet 2003; Wilhelmy y Durán, 2003; Quezada 2010; Portales 2011) señalan que entre los objetivos principales chilenos está la inserción económica del país, el posicionamiento como plataforma de exportaciones de Sudamérica al Asía Pacífico, la atracción de inversión extranjera y la búsqueda de mercados para productos elaborados a partir de un estado relativo más avanzado de industrialización, con destino particularmente en el cono sur y los países andinos.

Las prioridades de política exterior de Chile podrían resumirse en dos dimensiones. Primero, la promoción del libre comercio, la inserción económica y el multilateralismo; y, en segundo lugar, la promoción de la seguridad, la paz, la democracia y la protección a los derechos humanos (Fuentes, 2006). La coherencia de estos ejes de política exterior chilena desde 1990 hasta hoy es un rasgo destacado, pues los objetivos y las acciones de política se han mantenido consistentes. Sin embargo, pareciera ser que este hecho tiene su explicación en la continuidad de la misma coalición política durante viente años más que en grandes consensos nacionales en torno al tema. La debilidad de la política exterior chilena como una "política de Estado" se ha notado particularmente durante el reciente Gobierno de Sebastián Piñera, en el que Chile ha abandonado acuerdos internacionales en los que el país estaba participando o había promovido, tales como el acuerdo de Escazú y el Pacto Migratorio de Naciones Unidas. 
Para comprender el estado de la política exterior chilena a principios de los noventa no se puede ignorar el aislacionismo político que vivió el país durante la dictadura. De acuerdo a Fermandois (1991: 446), la inserción comercial que era parte de la estrategia económica del régimen de Pinochet fue un mecanismo que reemplazó la inserción política que había cobrado fuerza durante el Gobierno del presidente Frei Montalva en la segunda mitad de la década de los sesenta. Dicha estrategia se complementó una vez restaurada la democracia con una mayor acción política, especialmente en América Latina y en temas relacionados con la promoción de la democracia y los derechos humanos (Vargas 2012; Van Klaveren 2012). El aislamiento político del país significó que se perdiera la inserción internacional, y para lograr recuperarla el nuevo Gobierno debió generar un equipo ajeno al cuerpo diplomático, pero especializado para que asesorase al ministro, a la sazón Enrique Silva Cimma, carente también de experiencia internacional.

La transición a la democracia en Chile se caracterizó por la búsqueda del consenso, debido al sistema institucional heredado de la dictadura, así como por las características de la Concertación, que agrupaba desde la izquierda socialista que gobernó con Allende hasta la Democracia Cristiana. La política exterior no estuvo ajena a este fenómeno y de ahí que los objetivos que se trazaron al principio del Gobierno se ajustaran a esos mismos acuerdos. Sin embargo, se produjeron discusiones respecto del rol que debería jugar la Cancillería en este nuevo escenario en el cual conviviría una apertura comercial creciente con la búsqueda de la inserción política. En 1992, el ministro de Economía, Carlos Ominami, propuso crear un ministerio que se encargara exclusivamente de las relaciones económicas internacionales o bien que la Cancillería fuera reestructurada para que pudiese encargarse de mejor forma de los asuntos comerciales.

Frente a esto se deben destacar dos elementos: el primero es la forma de manejar los asuntos comerciales, a través de un comité de coordinación económica formado por la Cancillería, el Ministerio de Economía y el Ministerio de Hacienda, lo que es significativo pues revela que la actividad internacional no estuvo centralizada ni coordinada por la Cancillería. Lo segundo, es la relevancia que dentro de la Cancillería ha adquirido la política comercial, con su propio mecanismo de promoción internacional a través de PROCHILE. Estas agencias no están integradas por funcionarios de la carrera diplomática, y aunque el modelo de PROCHILE no es exactamente igual al de una misión diplomática, cumplen un rol esencial en el marco de la estrategia de política exterior del país. El ministro Carlos Ominami señaló que «debido a que las relaciones internacionales tienen cada vez un mayor componente económico y a veces se enfrenta una lógica más política de la Cancillería con una lógica más económica de las carteras de Economía y Hacienda y se produce además descoordinación nuestra con el sector privado [...] tampoco está claro que el poder de decisión internacional esté radicado en un ministerio y [además] el sector privado busca influir en las distintas

2. El Mercurio, «Hacia la diplomacia económica». Nota de prensa aparecida el 9 de agosto de 1992. 
carteras y se cruzan las líneas». La misma nota de prensa sostiene que la negociación de los acuerdos comerciales se encontraba parcelada: Hacienda encargándose del acuerdo con los Estados Unidos y México, mientras que Economía lideraba las negociaciones con Argentina, Bolivia, Venezuela y Europa. A ello se sumaba la existencia de comités de ministros, de subsecretarios y la presencia de la DIRECON y sus oficinas de PROCHILE.

Dentro de las críticas tradicionales a la Cancillería chilena se encuentran su nivel de profesionalización, lo que se nota especialmente en la proporción entre embajadores de carrera y embajadores nombrados por el presidente. La propia ley que regula el Servicio Exterior indica que aquellos funcionarios con rango de embajador son de exclusiva confianza del presidente. En una entrevista dada por la canciller Soledad Alvear $^{3}$ menciona que es necesario encontrar una justa combinación entre embajadores provenientes de la carrera diplomática y aquellos nombrados por el presidente, pues además de entenderse con el embajador uno tiene que entenderse con un asesor del presidente. Entonces se duplica el esfuerzo y no es tan eficiente. Esto es otro indicador de la preminencia del presidente en la formulación de la política exterior de Chile.

La Alianza del Pacífico permite ilustrar los rasgos del funcionamiento de la Cancillería chilena y la preminencia de la función comercial por sobre la política. Entre los elementos que han caracterizado a la acción de la Cancillería está la organización de seminarios para promocionar el acuerdo entre inversionistas interesados. En ese sentido, DIRECON ha ganado especial protagonismo en cuanto a la implementación de los acuerdos; sin embargo, en el proceso de discusión de la política la Cancillería no ha centralizado ni coordinado la agenda.

Las dimensiones normativas sobre la política burocrática permiten enmarcar el funcionamiento del MRE chileno. En la dimensión reality testing la pregunta es si la Cancillería puede centralizar la agenda. Frente a esto, la evidencia sugiere que no. Si bien hoy, especialmente en cuanto a la Alianza del Pacífico, la Cancillería ha aumentado su capacidad de coordinar la política comercial frente a la concurrencia de otros actores en comparación a lo que ocurría a principios de los noventa, la preminencia sigue siendo del presidente, mientras que otros ministerios participan activamente en la discusión de la estrategia internacional: Hacienda, Economía o Trabajo. La figura presidencial, en este caso, permite evitar algún tipo de confrontación burocrática mayor, especialmente cuando el presidente se ha hecho parte activa de la política internacional. Es decir, si bien varios actores en el Estado confluyen en la formulación de la política exterior del país, es el presidente el principal actor, por lo que el Ministerio tiene una capacidad limitada para definir el curso de la política exterior, lo que ocurre también con los otros actores interesados. Dicho de otra forma, en Chile la política

3. Raquel Correa, «La Soledad del poder. No me interesa ser candidata presidencial en 2005». Entrevista aparecida en El Mercurio el 1 de octubre de 2000. Sin embargo, el tema fue de extenso debate durante el Gobierno del presidente Lagos. Véase, por ejemplo, «Ruido de Embajadores" ${ }_{2}$ aparecido en El Mercurio el 24 de marzo de 2002. 
exterior se piensa en La Moneda y es plausible suponer que la consistencia de la agenda de política exterior chilena se explique por la permanencia de la misma coalición política entre 1990 y 2010 y no por la capacidad del MRE para actuar como un continuador de las ideas. Se ha generado así la percepción de que en Chile el Gobierno no es una variable relevante para explicar la estrategia de política exterior.

La segunda dimensión, acceptability, puede ser analizada también desde el rol del presidente y del MRE, y la pregunta es si la Cancillería puede sacar adelante una agenda sin buscar el compromiso de otros actores. Si el primer mandatario es excluido del análisis, el escenario es cercano a la formación de compromisos entre actores burocráticos, debido a la relación que la Cancillería debe emprender con otros actores en el Poder Ejecutivo, especialmente los ministerios del área económica. En el área comercial hoy la agenda tiende a converger y el MRE ha ganado experiencia en la materia, lo que le permite actuar con mayor autoridad en comparación a lo que ocurría a principios de los noventa, actuando así como un agente coordinador.

Sin embargo, al incorporar al análisis al presidente el escenario cambia. El presidente en Chile goza de una autoridad significativa para sacar adelante una agenda de política exterior sin buscar muchos compromisos con otros actores dentro de la burocracia. En ese sentido, el jefe de Gobierno actúa como un Foreign Policy Executive, decidiendo los cursos de acción y disponiendo de gran autonomía para formularlos. En consecuencia, incluso si el MRE generase una agenda de política exterior, el aval presidencial es esencial para llevarla a cabo. Así ocurrió, por ejemplo, con la reforma que se llevó a cabo sobre el sistema de cooperación internacional del país, cuando la AGCID (Agencia de Cooperación Internacional para el Desarrollo) pasó a formar parte de la estructura de la Cancillería, pero esto ocurrió solamente una vez que el presidente Lagos se hizo parte de la discusión respecto a la cooperación internacional de Chile.

La dimensión efficiency es probablemente en la que el MRE chileno está más cerca del punto óptimo, bajo el cual las decisiones se toman sin que se mantengan indefinidamente rotando entre diversos actores, pero tampoco sin apresurarse. Pero una vez más la alta influencia que tiene el presidente permite explicar esta situación, pues el decisor es claro y, además, ocurre que el desarrollo de la política exterior en los últimos años ha tendido a limitar las opciones entre los actores burocráticos y políticos debido a la consolidación de un consenso en torno a la inserción internacional del país. En el caso de la Alianza del Pacífico el tema resulta claro, ya que los objetivos de este bloque están en la misma frecuencia que los que Chile ha sostenido desde 1990.

\section{Politica exterior de Brasil y el rol de Itamaraty hacia Sudamérica}

Desde épocas muy tempranas del siglo xx, Brasil buscó posicionarse como un actor relevante en el escenario global, por lo que históricamente las acciones de política exterior brasileñas han estado orientadas por esta premisa de actuar como un gran país y por el deseo de influir en las relaciones políticas internacionales (Soares de Lima y Hirst, 2006). Vigevani y Cepaluni (2012) señalan que desde el Estado Novo, 
implementado por Getulio Vargas a mediados de los años treinta del pasado siglo, es posible identificar tres estrategias de política exterior para Brasil. La primera, denominada autonomía mediante distancia, se mantuvo hasta el segunda mitad de los ańos ochenta, se basaba en la distancia de los grandes centros de poder y respondía a una forma de desarrollo autárquica, ambas derivadas tanto del estructuralismo como del modelo de importación por sustitución de importaciones.

La autonomía mediante participación es la segunda estrategia y significó para Brasil hacerse parte de ciertas instituciones internacionales, como las rondas de negociación del GATT, y la aceptación de ciertas concepciones liberales del sistema internacional, lo que implicaba abandonar la industrialización por sustitución de importaciones. Sostener la estrategia de autonomía mediante distancia se habría traducido en un creciente aislacionismo, lo que no satisfaría los objetivos de largo plazo de la inserción internacional de Brasil.

La mayor apertura de Brasil después de la democratización iniciada en 1985 vino acompañada también de un mayor acercamiento hacia la región (Tickner, 2012; Malamud, 2012), específicamente hacia Paraguay, Argentina y, en menor grado, Venezuela. El caso con Argentina resulta interesante, pues tiene implicaciones en la seguridad regional (Sotomayor, 2004), ya que Brasil y Argentina rivalizaban por el liderazgo sudamericano y basaban su relación en la desconfianza, lo cual se manifestó en las divergencias por el uso de las aguas del río Paraná para fines hidroeléctricos, situación resuelta en 1979 con un tratado tripartito que incorporó a Paraguay; y en segundo lugar, la cuestión nuclear derivada de la capacidad que ambos países tienen para el desarrollo de energía atómica (Oelsner, 2005: 134)

Progresivamente en la agenda de política exterior brasileńa se fue consolidando una posición hacia los países del Mercosur, pero fue Cardoso quien promovió la profundización de las relaciones con otros países de América del Sur (Soares de Lima y Hirst, 2006: 30; Gomes Saraiva, 2010a). El lanzamiento de la Iniciativa de Infraestructura Regional para Sudamérica (IIRSA) el año 2000, fue el origen de un proceso de integración regional que incorporase a todos los países sudamericanos, pero cuyos fundamentos estaban en la relación construida previamente a través de Mercosur (Burges, 2008: 76).

La promoción de América del Sur como plataforma que incluye a Mercosur partió a mediados de los años noventa (Vigevani et al., 2003) y buscaba construir una esfera de influencia en una región en la que la dependencia con los Estados Unidos es menor, a diferencia de lo que ocurre en los países centroamericanos y caribeños, excluyendo también a México (Malamud, 2012: 169).

Además de Mercosur e IIRSA, Brasil impulsó para Sudamérica la creación de la UNASUR — primero Comunidad Sudamericana de Naciones - y del Consejo de Defensa Sudamericano con el objetivo de expandir su poder basado en la agregación de peso geopolítico de la región y su capacidad de negociación en el escenario internacional (Brands, 2011: 34; Soares de Lima y Hirst, 2006: 31), institucionalizando así sus aspiraciones de influencia a través de un mecanismo regional. Sin embargo, asumir el liderazgo regional no ha sido una tarea sencilla para Brasil. Por un lado, en el país 
los problemas regionales no son relevantes debido a la dimensión que adquieren los problemas domésticos debido a las características continentales de Brasil y, además, los asuntos globales, como medio ambiente y comercio, han adquirido una creciente importancia, especialmente durante los dos Gobiernos de Lula (Vigevani y Cepaluni, 2012). Esto conduce a que Brasil no actúe necesariamente como un paymaster en el proceso de integración en la región, algo particularmente necesario en un contexto de alta asimetría entre los Estados miembros (Mattli, 1999).

Durante los Gobiernos de Lula, Brasil empezó a adoptar una nueva estrategia denominada autonomía mediante diversificación, que consiste en profundizar relaciones con socios no tradicionales (Vigevani y Cepaluni, 2012). Ahí aparecen los países africanos, especialmente los de la Comunidad de Países de Lengua Portuguesa, pero también con Sudáfrica y Nigeria. Aprovechando el apelativo de «BRIC», la política exterior buscó establecer lazos con países emergentes que tuviesen características compartidas con Brasil: dimensiones continentales, reconocida importancia regional, población, producto interno bruto, recursos naturales, así como también intereses en un reordenamiento del sistema internacional (Gomes Saraiva, 2010b: 49). El objetivo de la autonomía mediante diversificación es funcionar como un puente entre el mundo en desarrollo - sin una pertenencia regional completamente definida - mundo desarrollado, construyendo lazos con países africanos y coaliciones con otros emergentes (Burges, 2013).

Varias características hacen que Itamaraty sea una de las Cancillerías más influyentes del mundo tanto en la implementación como en la formulación de la agenda de política exterior. La racionalización y la burocratización de la carrera diplomática ha sido uno de los elementos principales de la evolución de Itamaraty. Cheibub (1989) sostiene que Itamaraty avanzó en un proceso de creciente institucionalización, comparable solo con lo ocurrido dentro del Ejército, lo que dio lugar a una conciencia institucional dentro del Ministerio que le ha dotado de autonomía y lo ha tendido a aislar del resto de las agencias burocráticas. Esto genera ciertas ventajas, como la posibilidad de darle continuidad a la política exterior de Brasil, pero también desventajas, como la posibilidad de que Itamaraty goce de cierta autonomía en su función administrativa y quede alejado del control social y la rendición de cuentas (Cheibub, 1989: 126). Definido de esta forma, el MRE puede ser un actor político, por lo que se ajusta al modelo de política burocrática y permite analizar en qué medida las divergencias entre Planalto e Itamaraty pueden explicar los obstáculos en la implementación de la política exterior. No obstante, el propio Cheibub señala que si bien es necesario que Itamaraty conserve su centralidad en la política exterior, es deseable que otros actores puedan contrabalancear su influencia adquiriendo algún grado de institucionalización, de tal suerte que lleguen a ser grupos duraderos y estables (Cheibub, 1989: 127)

Al llegar los años noventa existía una visión respecto a que el presidente habría ganado relevancia en la política exterior, en detrimento de lo que hasta ese momento venía realizando el Ministerio (Sallum, 2011). El embajador Paulo Nogueira Batista indica que Itamaraty no tuvo una participación activa en la formulación de la política exterior brasileña durante el Gobierno de Collor de Mello, ni tampoco fue su principal 
ejecutor, a pesar que gracias al profesionalismo de sus cuadros diplomáticos pudo minimizar ciertas posturas presidenciales en aquellas políticas que debió implementar (Batista, 2009: 211).

Sin embargo, contrariamente a lo que afirma Batista, Stolle Paixão e Casarões (2012) señala que hay dos razones por las cuales la Cancillería ha sido un actor central en la política exterior brasileńa. En primer lugar, en un contexto democrático no son muchos los medios que se poseen para alejar a una burocracia del proceso decisorio y eso es particularmente difícil en la política exterior, pues su burocracia, responsable por su formulación e implementación, se ha visto sometida a un proceso de aislamien to burocrático. Y, en segundo lugar, porque las instrucciones entregadas por el presidente no eran necesariamente incompatibles con las líneas maestras que la política internacional había sostenido históricamente (Stolle Paixão e Ca saróes 2012, 137).

Gomes Saraiva (2010a) apunta a la existencia de dos grupos dentro de Itamaraty. Los institucionalistas pragmáticos o liberales y los autonomistas o nacionalistas. Los primeros se fortalecieron durante el Gobierno de Fernando Henrique Cardoso y favorecen una liberalización condicionada al priorizar el apoyo de Brasil a los regímenes internacionales desde una estrategia pragmática. Se trata de la defensa de ciertos valores universales, como la democracia y los derechos humanos, y la promoción de regímenes que permitan garantizarlos. El segundo grupo — los autonomistas - están ligados más a una visión desarrollista y, si bien no son originarios del PT, establecieron una relación significativa con el presidente Lula Da Silva. Este grupo le dio mayor énfasis a la autonomía del país y al fortalecimiento de la presencia brasileña en la política mundial. En ese contexto cobraba relevancia un esfuerzo sistemático para que Brasil adquiriese el liderazgo regional y pudiera incrementar así su ascenso como potencia global (Gomes Saraiva, 2010b: 49)

En suma, un alto nivel de profesionalización en Brasil le ha entregado al cuerpo diplomático una serie de competencias y habilidades para actuar en el planteamiento de la política exterior. El proceso de institucionalización ha aislado al Ministerio del resto de la Administración, lo que generó una fuerte conciencia institucional respecto al rol del país, y por mucho tiempo no hubo un contrapeso a la agenda alojada dentro del Ministerio, lo que se traduce en una alta capacidad para excluir a otros actores del proceso de formulación de la política.

La agenda de integración con Sudamérica venía siendo planteada desde el Ministerio desde principios de los noventa, principalmente hacia el Mercosur, y progresivamente cobró fuerza hacia fines del segundo Gobierno de Cardoso, por lo que adquirió relevancia la idea de la región sudamericana, pero principalmente con un carácter económico, enmarcado en Mercosur e IIRSA y basándose en las ideas institucionalistas. Durante el Gobierno de Lula Da Silva la región se consolida como una zona prioritaria para la política exterior de Brasil y gana relevancia dentro del Ministerio aquellas posturas más cercanas a la posición autonomista, sostenida por el PT. De esa forma se abrieron divergencias respecto a la estrategia de política adoptada y, especialmente, respecto al rol del asesor de relaciones internacionales de la Presidencia. 
A la hora de ubicar al MRE de Brasil en cada una de las dimensiones normativas de la política burocrática para el caso de UNASUR podemos decir que, respecto a la dimensión reality testing, el Ministerio se encuentra más cerca de la sobresimplificación que de la deliberación, pues mantiene una poder significativo para enmarcar la política dentro de su propia agenda, aunque no es totalmente capaz de cerrar la discusión a argumentos de otros actores, especialmente de la Presidencia y la sociedad civil. En la dimensión acceptability, el Ministerio está próximo al aislacionismo, pues Itamaraty es capaz de promover su agenda internacional sin buscar el apoyo de otros actores; sin embargo, el rol que ha venido jugando la Presidencia, especialmente durante los anteriores Gobiernos de Lula Da Silva, generaron presiones hacia una búsqueda mayor de compromisos. Con todo, la agenda de política externa sigue quedando en manos del MRE, a pesar del mayor rol que ha venido jugando, por ejemplo, el Banco Nacional de Desarrollo (BNDES) o el Ministerio de Industria y Comercio. Finalmente, en cuando a la dimensión efficiency, es plausible señalar que respecto a UNASUR el MRE se encontró más cerca de la economía burocrática que de los extremos y eso se podría explicar debido a la profesionalización del cuerpo burocrático y también a la existencia de una estrategia subyacente de política exterior que permite canalizar las decisiones de política pública de una forma eficiente.

\section{DISCUSIÓN COMPARADA}

Una vez revisado los dos casos, es posible sostener que dos son también los factores que permitirían explicar el rol que juega el MRE durante el proceso de formulación de política exterior y que diferencian lo que sucede en Brasil y Chile. Primero, el nivel de aislamiento de la Cancillería dentro de la Administración pública; segundo, su capacidad para coordinar centralizadamente la agenda de política exterior, lo que reduce el rango de preferencias posibles para los otros actores que se involucran en la discusión.

El MRE de Brasil ha ido consolidando a lo largo de los años no solo un Servicio Exterior profesional, sino además se ha ido diferenciando del resto de la Administración federal brasileña en cuanto a su nivel de institucionalización. Eso lo ha aislado del resto de los actores burocráticos en el Poder Ejecutivo y gracias a ello puede actuar con mucha más autonomía durante la formulación de la política. En Chile ocurre algo diferente, pues otros actores se han venido involucrando en el proceso de formulación de la política exterior y se han generado instancias formales o informales a través de las cuales la Cancillería se relaciona con otros actores dentro del Gobierno y los temas de política que se discuten terminan incluyendo las preferencias de varios actores.

La capacidad para coordinar centralizadamente la agenda de política exterior es otro elemento diferenciador y puede ser la variable más importante a la hora de explicar el rol de la Cancillería. El MRE de Brasil tiene la capacidad para centralizar y coordinar la agenda de política exterior, lo que reduce la información y las opciones disponibles dentro de su propio rango de preferencias. Eso no necesariamente tiene 
que ver con el grado de aislamiento, pues aun cuando puede existir relación con otros actores dentro del Poder Ejecutivo, la agenda la plantea el MRE y el resto de los actores debe sintonizar con ella. Solo en la última década, especialmente durante los Gobiernos del PT, la Presidencia ha contrapesado la agenda que plantea Itamaraty.

En Chile ocurre algo distinto, ya que son varios los ministerios con agendas internacionales, dada la dimensión comercial y política de las relaciones exteriores chilenas. Aparecen así, además de la Cancillería, los ministerios de Economía, Hacienda, Defensa e incluso el de Trabajo. De esa forma, la Cancillería no tiene preminencia sobre la agenda de política exterior y los temas que se discuten provienen de varios actores. En segundo lugar, la figura del presidente de la República es preponderante durante el proceso de política pública (no sólo en relaciones exteriores) y, por lo tanto, no solo juega un rol como decisor, sino que puede involucrarse activamente durante la formulación de la política exterior.

La profesionalización, si bien es relevante, no parece ser una variable suficiente para explicar el rol del MRE. Sí puede ser una variable que cobra fuerza en presencia de las otras dos variables antedichas. Es decir, un ministerio aislado $\left(\mathrm{X}_{1}\right)$ y con una gran capacidad para centralizar la agenda de política exterior $\left(\mathrm{X}_{2}\right)$ se verá beneficiado si cuenta con un servicio exterior profesional $\left(\mathrm{X}_{3}\right)$, pero si $\mathrm{X}_{1}$ y $\mathrm{X}_{2}$ están ausentes, la variable profesionalización es menos significativa para explicar el rol de la Cancillería en la formulación de la política exterior.

De ahí entonces que en Brasil el MRE tenga una capacidad creciente para generar un consenso burocrático en torno a la política exterior, mientras que en Chile quien tiene esa capacidad es el presidente. Dado el escenario que hemos analizado, resulta plausible señalar que en ausencia de un liderazgo presidencial fuerte en Chile, se tienda a una confrontación entre las agencias burocráticas involucradas en política exterior.

\section{CONCLUSIONES}

El objetivo de este trabajo ha sido estudiar el rol que ha jugado el MRE en la formulación de las estrategias de política exterior que han seguido Chile y Brasil en las últimas décadas, especialmente después de la democratización. Ambos países han mantenido una estrategia internacional consistente y la literatura sugiere que la continuidad estaría dada por un rol preponderante de la burocracia del Servicio Exterior, capaz de conservar las ideas que sustentan la política internacional y de esa forma aislarla de los ciclos políticos. Es decir, una Cancillería que juegue un rol preponderante en la formulación de política exterior puede influenciar la agenda, pues va desarrollando sus propias ideas respecto a la inserción internacional del país.

Para analizar cada ministerio se tuvieron en cuenta tres variables: la profesionalización, el nivel de aislamiento o inserción en la Administración pública y la capacidad para centralizar la agenda de política exterior. Asimismo, se usaron tres dimensiones normativas para estudiar la confrontación, deliberación o consenso burocrático respecto a la agenda de política exterior dentro de la Administración pública. En un 
escenario de consenso burocrático, el MRE sería un actor suficiente para llevar adelante la política, sin necesidad de buscar compromisos ni aceptar otros argumentos, mientras que ocurre lo opuesto en un escenario de confrontación, pues ningún actor es capaz de desarrollar una agenda de política y por lo tanto se produce una parálisis en torno al tema de política pública.

La evidencia sugiere que la variable más relevante para explicar el rol del MRE sería la capacidad que tiene para coordinar centralizadamente la agenda de política exterior. Eso no quiere decir que otros actores hayan sido excluidos, sino que los temas de política exterior pasen siempre por la Cancillería. En el caso brasileño parece claro que los asuntos internacionales son manejados por Itamaraty, aun cuando otros actores también pueden ser relevantes, tales como el BNDES, el Ministerio de Comercio, Petrobras e incluso el presidente. Sin embargo, las acciones internacionales de esos actores deben dialogar con la estrategia que emana de la Cancillería. En Chile, el MRE carece esa capacidad, a pesar de que en los ańos recientes ha venido ganando experiencia en el manejo de los asuntos comerciales. Quien sí posee esa capacidad es el presidente quien, por ende, es el actor central del proceso de formulación de la política exterior en Chile.

La profesionalización del MRE sería una variable necesaria, pero no suficiente para explicar un rol preponderante en la formulación de la política. Un ministerio con alta capacidad para centralizar la agenda de política exterior puede ver reforzado su rol en la medida en que cuente con un equipo profesional, como ocurre en Brasil. En el caso chileno, el MRE cuenta con niveles de profesionalización aún en proceso de consolidación, pero es plausible señalar que manteniendo un presidencialismo fuerte en la formulación de la agenda de política se podría conseguir una implementación más prolija e incluso algún nivel de influencia durante ese proceso, pero no necesariamente un contrapeso al presidente durante la formulación.

El nivel de aislamiento o inserción en la Administración puede ser un factor relevante para explicar el tipo de agendas de política que puede manejar el MRE. En el caso brasileño, el aislamiento del Ministerio dentro de la Administración le permite desarrollar agendas particulares, y dado que pueden centralizar la discusión de política exterior los otros actores, terminan actuando en sintonía o, al menos, sin divergir completamente de lo que propone Itamaraty. Ello ocurre incluso en el ámbito de la defensa. En el caso chileno el MRE no está aislado; por el contrario, existen y han existido mecanismos institucionales formales o informales que lo han relacionado con otros ministerios y con el presidente.

Es plausible concluir que la profesionalización del MRE es una variable que refuerza la posibilidad de la Cancillería para tener un rol relevante en la formulación de la agenda de política, pero solo si la Cancillería cuenta con la capacidad de centralizar la agenda de política exterior. Dicho de una forma más general, aquel actor que pueda centralizar la formación de la agenda de política exterior, sea la Cancillería, el presidente $\mathrm{u}$ otro ministerio — por ejemplo, Defensa - tendrá una probabilidad mayor de jugar un rol más relevante durante la formulación de la política en detrimento de otros y eso porque podrá reducir los argumentos y la información a asuntos de su preferencia, lo que reducirá así el conjunto de decisiones posibles. 
Algunas líneas que quedan pendientes a partir de este trabajo son: primero, analizar qué ocurre en otros casos de presidencialismo fuerte en la región, como Colombia o México, o allí donde se reconoce una influencia mayor del MRE, como Perú, así como ampliar el abanico de estrategias bajo análisis; en segundo lugar, analizar lo que ocurre dentro de los ministerios y como se organizan distintos grupos -en el caso de Brasil se ha visto la existencia de dos visiones sobre la estrategia internacional del país, y en el caso de Chile una división entre el Servicio Exterior y DIRECON—; en tercer lugar, buscar las razones que explican la capacidad de un ministerio para centralizar la agenda - pueden ser temas específicos, validación institucional o incluso podría explicarse por la propia formación del Estado y los procesos de racionalización y burocratización dentro de la Administración pública-. Finalmente, habría que analizar situaciones específicas de política a través de modelamiento de juegos, de manera tal que se pueda identificar la forma en la que se distribuyen las preferencias y la información entre los actores políticos, burocráticos y sociales, y también el rol que juega cada uno de ellos y cómo se alcanzan win sets favorables en determinados escenarios.

\section{AGRADECIMIENTOS}

El autor agradece los valiosos comentarios de los revisores anónimos de la RECP, así como también a Jaime Baeza Freer, profesor del Instituto de Asuntos Públicos de la Universidad de Chile, quien guió esta investigación.

\section{Referencias}

Alden, Cris y Amnon Aran. 2012. Foreign policy analysis: new approaches. Abingdon: Routledge.

Batista, Paulo Nogueira. 2009. "A Política Externa de Collor: Modernização ou Retrocesso?», en Pensando O Brasil. Ensaios e Palestras, de Paulo Nogueira Batista Jr. Brasilia: Fundação Alexandre de Gusmão.

Beasley, Ryan, Juliet Kaarbo, Charles Hermann y Margaret Hermann. 2001. «People and Processes in Foreign Policymaking: Insights from Comparative Case Studies», International Studies Review, 3 (2): 217-250. Disponible en: https://doi. org/10.1111/1521-9488.00238.

Brands, Hal. 2011. «Evaluating Brazilian Grand Strategy under Lula», Comparative Strategy, 30 (1): 28-49. Disponible en: https://doi.org/10.1080/01495933.2011. 545686.

Breuning, Marijke. 2007. Foreign Policy Analysis: A comparative introduction. Nueva York:Palgrave MacMillan.Disponible en: https://doi.org/10.1057/9780230609242.

Burges, Sean. 2008. "Consensual Hegemony: Theorizing Brazilian Foreign Policy after the Cold War», International Relations, 22 (1): 65-84. Disponible en: https:// doi.org/10.1177/0047117807087243. 
Burges, Sean. 2013. «Brazil as a bridge between old and new powers?», International Affairs 89 (3): 577-594. Disponible en: https://doi.org/10.1111/1468-2346.12034.

Carlsnaes, Walter. 2012. "Actors, structures and foreign policy analysis», en Steve Smith, Amelia Hadfield y Tim Dunne, Foreign policy: Theories, actors and cases. Oxford: Oxford University Press.

Cheibub, Zairo Borges. 1989. «A carreira diplomática no Brasil: O processo de burocratização do Itamarati», Revista de Administração Pública, 23 (2): 97-128.

Drezner, Daniel. (2000). «Ideas, bureaucratic politics and the crafting of foreign policy», American Journal of Political Science 44 (4): 733-749. Disponible en: https://doi.org/10.2307/2669278.

Fermandois, Joaquín. 1991. «De una inserción a otra: Política exterior de Chile 19661991», Estudios Internacionales 24 (96): 433-455.

Foyle, Douglas. 1997. «Public opinion and foreign policy: Elites beliefs as a mediating variable», International Studies Quarterly, 41(1): 141-169. Disponible en: https:// doi.org/10.1111/0020-8833.00036.

Fuentes, Claudio. 2006. «La apuesta por el poder blando: política exterior de la Concertación 2000- 2006", en Robert Funk. El gobierno de Ricardo Lagos: la nueva vía chilena hacia el socialismo. Santiago de Chile: Ediciones Universidad Diego Portales.

Gomes Saraiva, Miriam. 2010a. A diplomacia brasileira e as visóes sobre a inserção externa do Brasil: institucionalistas pragmáticos $x$ autonomistas. Madrid: Real Instituto Elcano; ARI. Disponible en: https://bit.ly/2HqmdVi.

Gomes Saraiva, Miriam. 2010b. «Brazilian foreign policy towards South America during the Lula administration: caught between South America and Mercosur", Revista Brasileira e Politica Internacional 53: 151-168. Disponible en: https://doi. org/10.1590/S0034-73292010000300009.

Gyngell, Alan y Michael Wesley. 2003. Making Australian Foreign Policy. Cambridge: Cambridge University Press. Disponible en: https://doi.org/10.1017/ CBO9780511755873.

Halperin, Morton, Priscilla Clapp, y Arnold Kanter. 2006. Bureaucratic politics and foreign policy. Washington D.C.: The Brookings Institution.

Hollis, Martin y Steve Smith. 1986. "Roles and reasons in Foreign Policy Decision Making», British Journal of Political Science 16 (3): 269-286. Disponible en: https://doi.org/10.1017/S0007123400004415.

Hudson, Valerie. 2005. "Foreign Policy Analysis: Actor-Specific Theory and the Ground of International Relations», Foreign Policy Analysis 1 (1): 1-30. Disponible en: https://doi.org/10.1111/j.1743-8594.2005.00001.x.

Hudson, Valerie. 2007. Foreign policy analysis: Classic and contemporary theory. Lanham, MD: Rowman and Littlefield Publishers.

Kaarbo, Juliet. 1997. "Prime Minister Leadership Styles in Foreign Policy Decision-Making: A Framework for Research», Political Psychology 18 (3): 553-581. Disponible en: https://doi.org/10.1111/0162-895X.00068. 
Kaarbo, Juliet. 1998. «Power Politics in Foreign Policy: The Influence of Bureaucratic Minorities", European Journal of International Relations 4 (1): 67-973. Disponible en: https://doi.org/10.1177/1354066198004001003.

Kaarbo, Juliet. 2001. «The domestic politics of international negotiations: The effects of states structures and policymaking process», International Interactions 24 (1): 169-205. Disponible en: https://doi.org/10.1080/03050620108434981.

Kaarbo, Juliet y Baris Kesgin. 2010. «When and How Parliaments Influence Foreign Policy: The Case of Turkey's Iraq Decision", International Studies Perspectives 11 (1): 19-36. Disponible en: https://doi.org/10.1111/j.1528-3585.2009.00390.x.

Kaarbo, Juliet y Deborah Grunfeld. 1998. «The social psychology of inter intra-group conflict in governmental politics», Mershon International Studies Review 42 (1): 226-233. Disponible en: https://doi.org/10.2307/254414.

Kaarbo, Juliet y Margaret Hermann. 1998. «Leadership styles of prime ministers: How individual differences affect the foreign policymaking process", The Leadership Quarterly 9 (3): 243-263. Disponible en: https://doi.org/10.1016/ S1048-9843(98)90029-7.

Keller, Jonathan y Dennis Foster. 2012. «Presidential Leadership Style and the Political Use of Force», Political Psychology 33 (5): 581-598. Disponible en: https://doi. org/10.1111/j.1467-9221.2012.00903.x.

Knecht, Thomas y Stephen Weatheford. 2006. «Public opinion and foreign policy: The stages of presidential decision making», International Studies Quarterly 50 (3): 705-727. Disponible en: https://doi.org/10.1111/j.1468-2478.2006.00421.x.

Mack, W., Karl DeRouen Jr, y David Lanoue. 2013. «Foreign Policy Votes and Presidential Support in Congress», Foreign Policy Analysis 9 (1), 79-102. Disponible en: https://doi.org/10.1111/j.1743-8594.2011.00166.x.

Malamud, Andrés. 2012. «Moving regions: Brazil's global emergence and the redefinition of Latin American borders», en Pía Riggirozzi y Diana Tussie, The rise of post-hegemonic regionalism. The case of Latin America. Nueva York: Springer. Disponible en: https://doi.org/10.1007/978-94-007-2694-9_9.

Marsh, Kevin. 2013. "Obama's Surge: A bureaucratic politics analysis of the decision to order a troop surge in the Afghanistan War», Foreign Policy Analysis 10 (3): 265288. Disponible en: https://doi.org/10.1111/fpa.12000.

Marshall, Bryan y Brandon Prins. 2011. «Power or posturing? Policy availability and congressional influence on U.S. Presidential decisions to use force», Presidential Studies Quarterly, 41 (3): 521-545. Disponible en: https://doi.org/10.1111/ j.1741-5705.2011.03885.x.

Mattli, Walter. 1999. The Logic of Regional Integration. Europe and Beyond. Nueva York: Cambridge University Press. Disponible en: https://doi.org/10.1017/ CBO9780511756238.

Milet, Paz. 2003. «La política exterior de los gobiernos de la Concertación», Colombia Internacional 56 (57), 47-63.

Oelsner, Andres. 2005. International Relations in Latin America. Peace and Security in the Southern Cone. Nueva York: Routledge. 
Pimenta de Faria, Carlos Aurélio, Dawisson Belém Lopez y Guilherme Casaroes. 2013. "Itamaraty on the move: Institutional and political change in Brazilian foreign service under Lula Da Silva's presidency (2003-2010)", Bulletin of Latin American research 32 (4): 468-482. Disponible en: https://doi.org/10.1111/ blar.12067.

Portales, Carlos. 2011. «Desafíos para la política exterior en las próximas décadas: Chile y la región en el mundo global», Estudios Internacionales 44 (169): 171-193.

Preston, Thomas y Paul 't Hart. 1999. "Understanding and evaluating bureaucratic politics: The nexus between political leaders and advisory systems», Political Psychology 20 (1): 49-98. Disponible en: https://doi.org/10.1111/0162-895X.00137.

Quezada, Abraham. 2010. «Inserción internacional de Chile en la post guerra fría: Concertación política e integración económica comercial; dos ejes conceptuales de la política exterior del gobierno de Ricardo Lagos (2000-2006)», Enfoques, 8 (13): 119-134.

Rosati, Jerel. 1981. «Developing a systematic decision-making framework. Bureaucratic politics in perspective», World Politics, 33 (2): 236-252. Disponible en: https:// doi.org/10.2307/2010371.

Sallum, Brasilio Jr. (2011). «Governo Collor: O Reformismo Liberal e a Nova Orientação da Política Externa Brasileira», DADOS: Revista de Ciências Sociais, 54 (2): 259-288. Disponible en: https://doi.org/10.1590/S0011-52582011000200002.

Soares de Lima, Maria Regina y Monica Hirst. 2006. «Brazil as intermediate state and regional power: action, choice and responsibilities», International Affairs, 82 (1): 21-40. Disponible en: https://doi.org/10.1111/j.1468-2346.2006.00513.x.

Sotomayor, Arturo. 2004. "Civil-Military affairs and security in the southern cone: The sources of Argentine-Brazilian Nuclear cooperation", Latin American Politics and Society, 46 (4): 29-60. Disponible en: https://doi.org/10.1353/lap.2004.0053.

Stolle Paixão y Casarôes, Guilherme. 2012. «O papel do Itamaraty na definição da política externa do governo Collor de Mello», Revista Brasileira de Politica Internacional, 55 (1): 135-153. Disponible en: https://doi.org/10.1590/S0034-73292012000100008.

Taliaferro, Jeffrey, Steven Lobell y Norrin Ripsman. 2009. «Introduction: Neoclassical realism, the state of the foreign policy», en Jeffrey Taliaferro, Steven Lobell y Norrin Ripsman (eds.), Neoclassical realism, the state and foreign policy. Nueva York: Cambridge University Press. Disponible en: https://doi.org/10.1017/ CBO9780511811869.001.

Tickner, Arlene. 2012. «Rising Brazil and South America», en Steven Smith, Amelia Hadfield y Tim Dunne (eds.), Foreign policy. Theories, actor, cases. Oxford: Oxford University Press.

Van Klaveren, Alberto. 2012. «La promoción de la democracia como principio de la política exterior de Chile», en Mario Artaza y César Ross, La política exterior de Chile, 1990-2009. Del aislamiento a la integración global. Santiago de Chile: RIL Editores. 
Vargas, Edmundo. 2012. «La política exterior de Chile en derechos humanos», en Mario Artaza y César Ross (eds.), La politica exterior de Chile, 1990-2009. Del aislamiento a la integración global. Santiago de Chile: RIL Editores.

Vigevani, Tullo y Gabriel Cepaluni. 2012. Brazilian foreign policy in changing times. The quest for autonomy from Sarney to Lula. Plymouth: Lexington Books.

Vigevani, Tullo, Marcelo De Oliveira y Rodrigo Cintra. 2003. "Política externa no período FHC: a busca de autonomia pela integração», Tempo Social 15 (2): 31-61. Disponible en: https://doi.org/10.1590/S0103-20702003000200003.

Welch, David. 1992. "The organizational process and bureaucratic politics», International Security, 17 (2): 112-146. Disponible en: https://doi.org/10.2307/2539170.

Wilhelmy, Manfred y Roberto Durán. 2003. «Los principales rasgos de la política exterior chilena entre 1973 y 2000», Revista de Ciencia Politica 23 (2): 273-286. Disponible en: https://doi.org/10.4067/S0718-090X2003000200014.

Wilhelmy, Manfred y Rosa Lazo. 1997. «La política multilateral de Chile en Asia-Pacífico», Estudios Internacionales, 30 (117): 3-35.

Presentado para evaluación: 23 de mayo de 2017.

Aceptado para publicación: 7 de marzo de 2019.

\section{PABLO VALENZUELA}

pablo.valenzuela@mail.com

Es licenciado en Ciencias Políticas y Gubernamentales y magíster en Ciencia Política por el Instituto de Asuntos Públicos de la Universidad de Chile. Actualmente trabaja como investigador de la Facultad de Humanidades de la Universidad de Santiago de Chile. Además, cursa el magíster en Desarrollo Urbano del Instituto de Estudios Urbanos y Territoriales de la Facultad de Arquitectura, Diseño y Estudios Urbanos de la Pontificia Universidad Católica de Chile. 